CASE REPORT

\author{
R.A. Salgado \\ P.G. Jorens \\ I. Baar \\ P. Cras \\ G. Hans \\ P.M. Parizel
}

\section{Methadone-Induced Toxic Leukoencephalopathy: MR Imaging and MR Proton Spectroscopy Findings}

SUMMARY: We report the clinical, MR imaging, and proton MR spectroscopy findings in a middle-aged woman with proved methadone-induced toxic leukoencephalopathy. The imaging characteristics of this unusual condition have been reported only rarely in the medical literature. We show that the imaging findings in methadone-induced toxic leukoencephalopathy are similar, though not identical, to previously reported cases of neurologic deterioration due to heroin inhalation.

ABBREVIATIONS: Cho $=$ choline; $\mathrm{Cr}=$ creatine; FLAIR = fluid-attenuated inversion recovery; NAA $=N$-acetylaspartate
T oxic leukoencephalopathy can be caused by a wide variety of agents, including occupational or environmental exposure, substance abuse, radiation, and chemotherapy. We report the clinical, MR imaging, and proton MR spectroscopy findings of a rare case of proved methadone-induced toxic leukoencephalopathy in a middle-aged woman. The related literature is also discussed.

\section{Case Report}

A 65-year-old woman was referred to a regional hospital with a deteriorating neurologic clinical condition. Clinical neurologic examination revealed apathy, a catatonic state with extreme rigidity, increased reflexes in the upper limbs, and a bilaterally positive Babinski sign. Laboratory investigation showed no signs of electrolyte disturbances, liver or kidney failure, inflammation, or other metabolic disturbances.

Previous medical history revealed placement of a hip prosthesis 12 months before the present admission. Since then the patient had chronic intractable pain treated with a neurostimulator and orally administered methadone $(3 \times 10 \mathrm{mg} /$ day $)$. However, 1 month before admission, she accidently took a higher methadone dose of $3 \times 30$ $\mathrm{mg}$ /day during an undetermined period due to an error of the pharmacologist.

Toxicology screening (urine and serum) revealed very high amounts of an opioid identified as methadone. No quantitative determination was undertaken.

Twenty-one days after the acute event, the patient was transferred to our hospital. On day 27 after the acute episode, an MR imaging examination of the brain was performed on a $1.5 \mathrm{~T}$ superconducting magnet. FLAIR and T2-weighted images showed extensive and symmetric signal-intensity abnormalities in the deep white matter of both cerebral hemispheres, with sparing of the subcortical U-fibers (Fig 1). The affected areas had very high signal intensity on diffusionweighted trace images, but without corresponding diffusion restric-

Received May 4, 2009; accepted after revision June 2

From the Departments of Radiology (R.A.S., P.M.P.), Critical Care Medicine (P.G.J.), Neurology and Born Bunge Institute Faculty of Medicine, (I.B., P.C.), and Multidisciplinary Pain Center (G.H.), Antwerp University Hospital, University of Antwerp, Edegem, Belgium.

Please address correspondence to Rodrigo A. Salgado, MD, Department of Radiology, Antwerp University Hospital, University of Antwerp, Wilrijkstraat 10, 2650 Edegem, Belgium; e-mail: rodrigo.salgado@uza.be or rodrigo.salgado@scarlet.be

DOI 10.3174/ajnr.A1889 tion on apparent diffusion coefficient maps (not shown). The posterior fossa structures were normal.

Proton single-voxel MR spectroscopy was performed by using TE values of 30,135 , and $270 \mathrm{~ms}$, consecutively. We found a marked decrease of the NAA peak with a relative increase of the choline peak (Fig 2). Furthermore, there was a lactate peak with a resonance frequency at $1.32 \mathrm{ppm}$.

On the basis of the MR imaging and proton MR spectroscopy findings, combined with a history of accidental ingestion of a high dose of methadone and the exclusion of exposure to other neurologically toxic substances, we established the diagnosis of methadoneinduced toxic leukoencephalopathy.

In the following month, the patient slowly recovered.

\section{Discussion}

Substitute drugs such as methadone are widely used both in medication-assisted therapy of drug abuse/dependence as well as in the management of intractable pain. Nevertheless, due to its increasing use and availability, methadone overdose has been reported as a growing problem. ${ }^{1}$

Most of the available literature on imaging of illicit substances deals with the neurotoxic changes induced by heroin, a morphine agonist, administered either intravenously or through (repeated) inhalation. ${ }^{2-7}$ There are only a few reported cases of severe methadone-induced toxic leukoencephalopathy that include imaging findings. ${ }^{8-10}$

The imaging findings in our patient are similar, though not identical, to previously reported MR imaging and spectroscopic white matter changes observed in heroin-vapor-inhalation toxicity (a practice known as "chasing the dragon"). ${ }^{2-4,6,7}$ In general, the different case reports mention similar symmetric and confluent high-signal-intensity changes in the cerebral white matter observed on T2-weighted and FLAIR images with, as also observed in our patient, sparing of the subcortical U-fibers. In our patient, there was no evidence of heroin abuse in any form or involvement of any other recreational drug.

However, most previously published reports on heroininduced neurotoxicity also mention white matter changes in the cerebellum and brain stem, which were absent in our patient. Furthermore, the few case reports with CT and MR imaging findings describing brain damage after a methadone overdose all reported cerebellar swelling with subsequent de- 

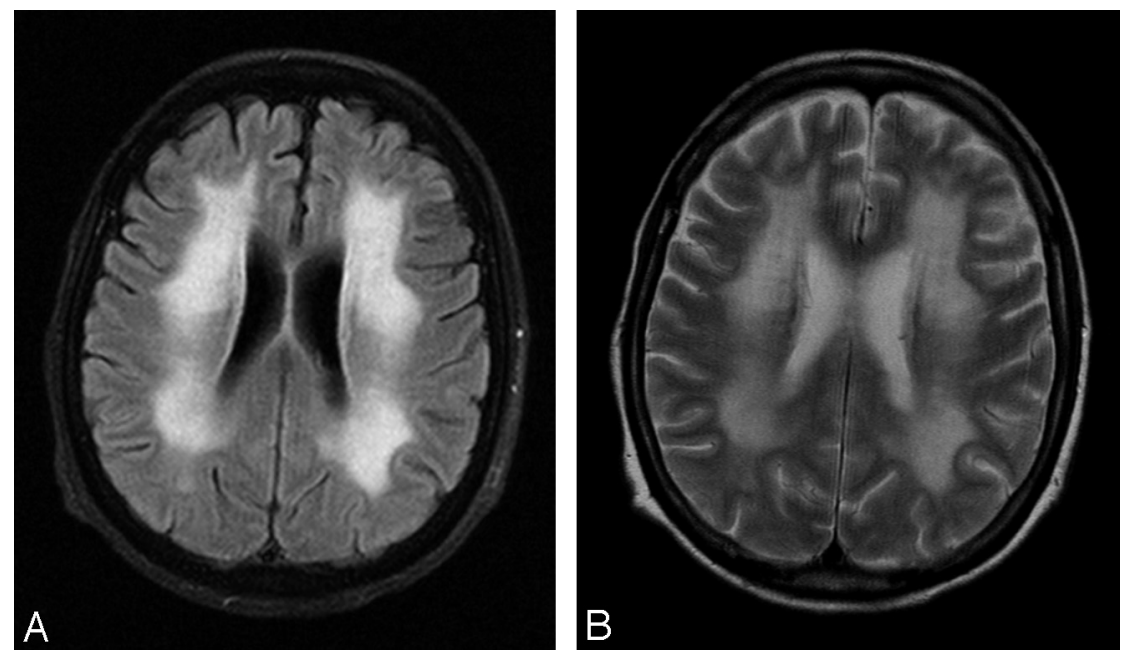

Fig 1. Axial FLAIR image with fat saturation $(A)$ and a turbo spin-echo T2-weighted image $(B)$. There are confluent and strikingly symmetric signal-intensity abnormalities in the white matter of both cerebral hemispheres, with sparing of the subcortical U-fibers. The posterior fossa structures are normal.

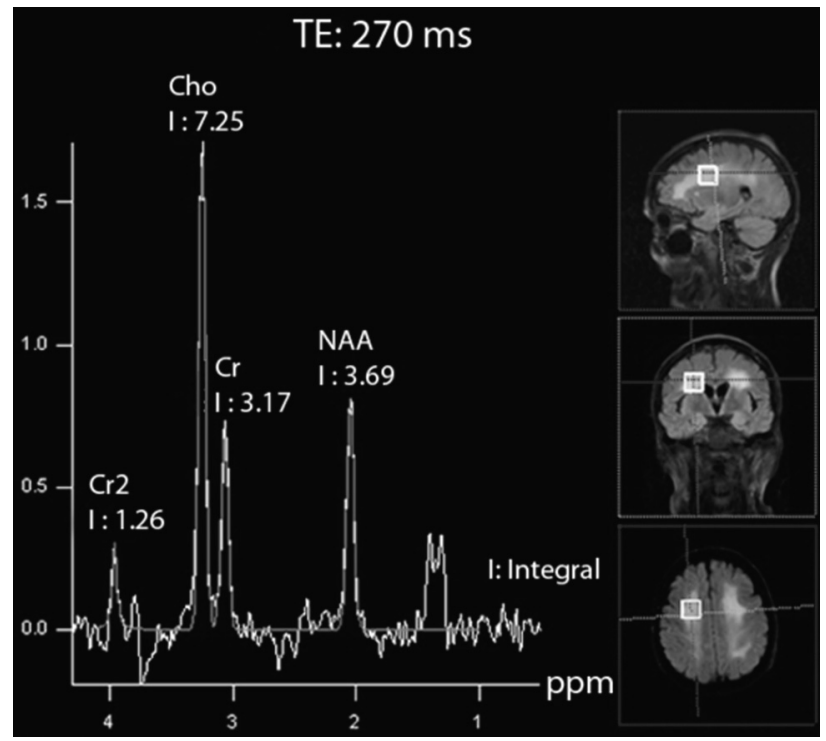

Fig 2. Proton single-voxel MR spectroscopy, performed with a TE value of $270 \mathrm{~ms}$. There is a striking decrease of the NAA peak with a relative increase of the choline peak. A lactate peak with a resonance frequency at $1.32 \mathrm{ppm}$ is present and shows a characteristic "up" doublet configuration.

velopment of acute hydrocephalus. ${ }^{8-10}$ In these reports, all the patients were children with a maximum age of 3 years. However, to the best of our knowledge, this is the first case report of methadone intoxication in an adult.

Some investigators have hypothesized that the neurotoxicity of heroin may be due to a contaminant or additive to the drug. ${ }^{4,11}$ However, in our patient, there was no such a component. Similarly, a previous study by Wolters et $\mathrm{al}^{11}$ failed to clearly identify a neurotoxic contaminant or additive responsible for the inflicted white matter damage after heroin inhalation. Furthermore, Nanan et al ${ }^{12}$ described white matter changes in a young girl after intoxication with morphine sulfate tablets, a noncontaminated pure morphine agonist, which could not be fully explained by a hypoxic-ischemic event.

Mitochondrial dysfunction and damage can explain the decrease in NAA and the increase in lactate. Choline is a marker in the synthesis and breakdown of cell membranes.
Lactate is a marker for anaerobic metabolism. The proton MR spectroscopy findings revealed a decreased NAA peak and an increase in the lactate peak, similar to findings in previous reports of inhaled heroin neurotoxicity. ${ }^{6,7}$ As stated by Vella et $\mathrm{al},{ }^{13}$ these findings may indicate a mixed pathologic process of hypoxia and mitochondrial damage, with axonal injury but without demyelination.

In conclusion, the MR imaging and proton MR spectroscopy findings of methadone-induced neurotoxicity in our patient are similar but not identical to those in previous reports of heroin-induced brain damage. Techniques like proton MR spectroscopy of the involved areas can be of assistance in further work-up. Additional studies are, nevertheless, needed to understand the underlying pathophysiology adequately.

\section{References}

1. Wolff K. Characterization of methadone overdose: clinical considerations and the scientific evidence. Ther Drug Monit 2002;24:457-70

2. Chen CY, Lee KW, Lee CC, et al. Heroin-induced spongiform leukoencephalopathy: value of diffusion MR imaging. J Comput Assist Tomogr 2000;24:735-37

3. Gacouin A, Lavoue S, Signouret T, et al. Reversible spongiform leucoencephalopathy after inhalation of heated heroin. Intensive Care Med 2003;29:1012-15. Epub 2003 Mar 14

4. Keogh CF, Andrews GT, Spacey SD, et al. Neuroimaging features of heroin inhalation toxicity: "chasing the dragon." $A J R A m$ J Roentgenol 2003;180:847-50

5. Kriegstein AR, Shungu DC, Millar WS, et al. Leukoencephalopathy and raised brain lactate from heroin vapor inhalation ("chasing the dragon"). Neurology 1999;53:1765-73

6. Offiah C, Hall E. Heroin-induced leukoencephalopathy: characterization using MRI, diffusion-weighted imaging, and MR spectroscopy. Clin Radiol 2008;63:146-52

7. Chang WC, Lo CP, Kao HW, et al. MRI features of spongiform leukoencephalopathy following heroin inhalation. Neurology 2006;67:504

8. Anselmo M, Campos Rainho A, do Carmo Vale M, et al. Methadone intoxication in a child: toxic encephalopathy? J Child Neurol 2006;21:618-20

9. Riascos R, Kumfa P, Rojas R, et al. Fatal methadone intoxication in a child Emerg Radiol 2008;15:67-70

10. Mills F, MacLennan SC, Devile CJ, et al. Severe cerebellitis following methadone poisoning. Pediatr Radiol 2008;38:227-29

11. Wolters EC, van Wijngaarden GK, Stam FC, et al. Leucoencephalopathy after inhaling "heroin" pyrolysate. Lancet 1982;2:1233-37

12. Nanan R, von Stockhausen HB, Petersen B, et al. Unusual pattern of leukoencephalopathy after morphine sulphate intoxication. Neuroradiology 2000;42:845-48

13. Vella S, Kreis R, Lovblad KO, et al. Acute leukoencephalopathy after inhalation of a single dose of heroin. Neuropediatrics 2003;34:100-04 